Abstract 24 Table 1 Descriptives on demographic, insomnia, and sleep factors ( $N=74)$

\begin{tabular}{llc}
\hline \multicolumn{1}{c}{$\mathbf{N}=\mathbf{7 4}$} & \multicolumn{1}{c}{ Pre-Confinement (\%/M(SD)) } & Confinement (\%/M(SD)) \\
\hline Gender (female) & \multicolumn{2}{c}{$68 \%$} \\
Age (years) & & $25.1(4.4)$ \\
Competition level (International) & & $92 \%$ \\
Insomnia symptoms & $75 \%$ & $49 \%$ \\
Insomnia disorder & $35 \%$ & $28 \%$ \\
Total sleep time (minutes) & $456(49.8) \mid 7 \mathrm{~h} 36 \mathrm{mins}$ & $490.8(69.7) \mid 8 \mathrm{~h} 10 \mathrm{mins}$ \\
Total time in bed (minutes) & $522.8(49.9) \mid 8 \mathrm{~h} 48 \mathrm{~min}$ & $574.3(71.9) \mid 9 \mathrm{~h} 34 \mathrm{~min}$ \\
Training load (minutes/daily) & $254(72.5) \mid 4 \mathrm{~h} 14 \mathrm{~min}$ & $196.8(81.4) \mid 3 \mathrm{~h} 16 \mathrm{~min}$ \\
\hline
\end{tabular}

potential athlete-specific sleep risk factors of training, competition, and travel.

Participants (competition level $\geq$ national) completed baseline (prior to 23rd March 2020) and home confinement (commenced 5th May 2020) assessments. The 10-section online survey included: the PSQI; MCTQ (Munich Chronotype Questionnaire); rMEQ (Reduced Morningness-Eveningness Questionnaire; FIRST (Ford Insomnia Response to Stress Tests); PSAS-C (Pre-Sleep Arousal Scale-C); and GAD-7 (Generalised Anxiety Disorder 7-item scale), with additional expertdesigned questions addressing sleep practices and DSM-5 insomnia symptoms.

Differences $(\mathrm{N}=74)$ between baseline and confinement responses were calculated with t-tests, Wilcoxon and $\mathrm{McNe}$ mar's tests. There was a reduction in prevalence of insomnia symptoms $(75 \%$ vs $49 \% ; \mathrm{p}=0.002)$ and insomnia disorder $(35 \%$ vs $28 \% ; p=0.286)$ during confinement. Increased during confinement was total sleep time $(7 \mathrm{~h} 36 \mathrm{~min}$ vs $8 \mathrm{~h} 10 \mathrm{~min}$; $\mathrm{p}<0.0001)$ and total time in bed before training days (8h48min vs 9 h34min; $\mathrm{p}<0.0001$ ). Training load (minutes/ daily) was reduced $(4 \mathrm{~h} 14 \mathrm{~min}$ vs $3 \mathrm{~h} 16 \mathrm{~min}$; $\mathrm{p}<0.0001)$ in confinement (table 1). 19\% of participants resolved pre-confinement insomnia disorder during confinement (figure 1).

During confinement, participants registered lowered prevalence of insomnia and training load. This research offers valuable insight on the insomnia profile of confined elite athletes, also addressing the role of the athletic lifestyle in insomnia prevalence.

\section{PILOTING MODIFIED COGNITIVE BEHAVIOURAL THERAPY FOR INSOMNIA (CBT-I) IN A COMMUNITY MENTAL HEALTH TEAM (CMHT)}

${ }^{1}$ Vinay Mandagere*, ${ }^{2}$ Phoebe Whishart, ${ }^{2}$ Jane Hicks. 'University of Bristol Medical School, Bristol, UK; ${ }^{2}$ Avon and Wiltshire Mental Health NHS Partnership Trust, Bristol, UK

\subsection{6/bmjresp-2021-bssconf.22}

Introduction Cognitive Behavioural Therapy for Insomnia (CBT-I) is the first line therapy for insomnia, a common risk factor in psychiatry. We investigated whether piloting a CBT-I programme within a Community Mental Health Team (CMHT) improves insomnia symptoms. Our programme consisted mainly of Sleep Restriction Therapy (SRT) determined by individual chronotypes. CBT-I is currently under-resourced in the UK. To our knowledge, this was the first NHS programme in Bristol secondary mental health.

Methods 10 participants were recruited. Participants underwent a therapist guided initiation on (a) Sleep education (b) Sleep hygiene (c) Stimulus Control. Individual sleep windows were determined by the participants' chronotype: whether they were a 'morning lark' or 'evening owl'. Participants then underwent a 6-week course of Sleep Restriction Therapy (SRT). Weekly follow up focussed around motivation and explanation was either by phone or face-to-face due to the COVID-19 pandemic. Outcome measurements used pre- and post-intervention sleep diaries; as well as insomnia, depression (PHQ-9) and general health questionnaires (SF-36).

Results There was little improvement in Total Sleep Time (TST) $(\mathrm{d}=-0.84$ hours) and patient-reported sleep quality $(\mathrm{d}=$ -0.67) following a 6-week course of modified CBT-I. Despite this, average number of mid-sleep awakenings roughly halved (47.9\%). ISI, PHQ-9 and SF-36 questionnaires demonstrated no difference between pre-intervention and post-intervention scores. Unstructured interviews revealed that patients' thoughts and anxieties at night-time interfered with SRT.

Conclusions Our study suggests that modified CBT-I is a challenge for mental health populations. Solely SRT may not be sufficient to treat insomnia secondary to mental illness. Treating co-morbid insomnia may therefore require multi-component CBT-I to address sleep-related mental health issues, such as panic attacks, flashbacks and nightmares. CBT-I in secondary mental health services requires further development, with long-term follow up of patients to evaluate adherence to the programme and the behavioural changes needed.

\section{RESPIRATORY ANNUAL REVIEW FOR CHILDREN \& YOUNG PEOPLE WITH NEUROMUSCULAR CONDITIONS AND COMPLEX NEURODISABILITY: A PILOT STUDY}

${ }^{1}$ Sairah Akbar, ${ }^{1}$ Ruth Wakeman*, ${ }^{1}$ Caroline Davies-Jones, ${ }^{1} J o a n n e$ Gregory, ${ }^{1}$ Alexander Thomas, ${ }^{1}$ Bernadette Ortega, ${ }^{2}$ Federica Trucco. ${ }^{1}$ Royal Brompton Hospital, Guys and St Thomas' NHS Foundation Trust, London, UK; ${ }^{2}$ Department of Paediatric Neuroscience, Guy's and St Thomas' NHS Foundation Trust and Department of Paediatric Respiratory Medicine, Royal Brompton Hospital, London, UK

\subsection{6/bmiresp-2021-bssconf.23}

Introduction Children with complex neurological and neuromuscular conditions often have respiratory involvement requiring ventilatory support and multidisciplinary expertise due to their complexity.

To ensure patients' needs are met whilst minimising repeated hospital visits, an Annual Review (A/R) pathway was created to combine sleep study and multi-professional review within one admission.

Method Patients under our care with a scheduled in-patient sleep study were offered a full $\mathrm{A} / \mathrm{R}$ as part of the pilot (August 2020-2021). Table 1 outlines investigations and reviews undertaken during $\mathrm{A} / \mathrm{R}$ (tailored to the individual). 